Moreover, the company says the biocides (bromo-nitro-dioxane, methylisothiazolone, hydroxypyridine-oxide, imidazolidinylurea and chloroacetamide) are mercury-free and are less toxic than sodium azide at working concentrations, as well as being neither carcinogenic nor mutagenic (Ames-test negative). Boehringer says that BM biocides exhibit little or no risk of assay interference, feature a low minimal concentration of inhibition, and do not discolor reagents. For convenience, a sampling kit has been developed that provides users with an economical way to evaluate the effectiveness of the biocides in their own reagents. The kit contains sample sizes of each biocide, in addition to information that can assist in their proper use and selection.

\section{Reader Service No. 94 \\ Tel. (+1) 317-576-2711 \\ Fax (+1) 317-576-7317}

Leica's Cryofect disinfectant spray zaps bacteria, fungi and viruses at temperatures as low as $-20^{\circ} \mathrm{C}$.

The Cryofect disinfectant spray from Leica is a virucidal and germicidal agent that is said to be effective against bacteria, fungi and viruses, such as hepatitis $B$ virus, HIV and polio. As such, it is designed to guard against the hazards of handling potentially infectious material. Leica says it can be applied at cryochamber temperatures down to $-20^{\circ} \mathrm{C}$, which means that the spray can be used even though the cryostat is not defrosted. Including incubation of the spray, the entire disinfecting process takes no more than $25 \mathrm{~min}$.

Reader Service No. 95

Tel. (+49) (0) 6224-143-113

Fax $(+49)(0) 6224-143-200$

\section{SAFETY GADGETS/EQUIPMENT}

The WaterSence automatic faucet controller from Coast Scientific is designed to protect patients and staff from crosscontamination and to eliminate fomites and vectors. Intended for use in hospitals and laboratories, and anywhere contamination is a consideration, the WaterSence device is controlled by an ultrasonic, computer-driven sensor that automatically controls water flow when the user's hands move under the faucet. Coast Scientific says no plumbing is required to install the device, which

screws onto the end of a faucet and requires one $9-\mathrm{V}$ battery for operation.

\title{
Reader Service No. 96
}

Tel. (+1) 619-450-0999

Fax $(+1) 619-450-1157$

The MicroMAT Class II safety cabinet from Medical Air Technology has been designed to house a high-powered microscope, ensuring a high level of protection for both the operator and the sample under test in microscopical procedures that require

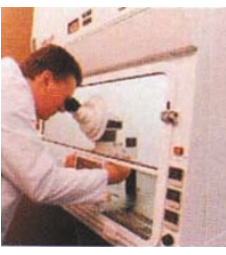

MicroMAT safety cabinet comfortably houses a high-powered microscope. aseptic conditions. The

cabinet has been designed around the microscope and features a specially shaped front screen that is said to provide maximum protection for the operator, allowing easy access and operation of the microscope and reducing the risk of microbial contamination.

\section{Reader Service No. 97}

Tel. $(+44)(0)$ 161-320-5652

Fax $(+44)(0) 161-335-0313$

The 5810 standard and 5810R refrigerated compact centrifuges from Eppendorf-Netheler-Hinz can accommodate up to 36 Falcon tubes of $15 \mathrm{ml}$ or 100 blood-collecting cups. After the rotor has been loaded, the motorized lid closes without requiring any force. Thirty-four run programmes are available to set the rotor to a speed of up to 14,000 r.p.m. With the refrigerated version, samples can be cooled to temperatures of between $-9{ }^{\circ} \mathrm{C}$ and $+40{ }^{\circ} \mathrm{C}$. In its swingbucket rotor, temperature-sensitive samples are kept at $+4{ }^{\circ} \mathrm{C}$, even at maximum speed, says the company. Other design features include an imbalance sensor, automatic rotor recognition, short-spin function and user-selectable speed, RCF or radius correction values. Several different swing-bucket and fixedangle rotors are available. The swingbucket rotor holds up to four 250-ml rectangular or microtiter plate buckets, which can be inserted directly. The modular design of the rectangular bucket adapters allows test tubes of varying lengths to be used. Moreover, the rectangular buckets can be sealed with aerosol-tight caps.

\section{Reader Service No. 98}

Tel. $(+49)(0)$ 4053-801-239

Fax (+49) (0) 4053-801-556

\section{STAINING SYSTEMS}

Software control provides a good user interface for the Leica ST 5050 automated immunostainer, which can be enhanced by the remote PC option. Multiple programmes can be run simulta-

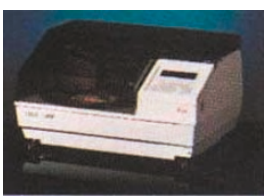

High throughput and software control - features of Leica's ST5050 automated immunostainer.

neously in any combination, including selectable spray positions. An integral printer port allows the documentation of all programme and staining parameters, including staining protocols. The pipettor system delivers a precise amount of reagent onto the user-defined slide position: Up to twenty different primary antibodies and up to sixteen additional reagents can be used per run. Moreover, the temperature can be adjusted from ambient up to $45^{\circ} \mathrm{C}$. Washing parameters, incubation times and the cleaning routine can also be programmed.

Reader Service No. 99

Tel. (+1) 847-405-0123

Fax (+1) 847-405-0147

Sigma's ready-to-use 3,3'-diaminobenzidine tetrahydrochloride, or DAB, liquid enzyme substrate systems are designed to eliminate reagent preparation and variation in immunohistological and im-

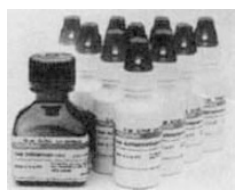

Sigma's DAB liquid enzyme substrate comes ready for use. munoblotting procedures. The kits contain premeasured and premixed volumes of reagents for the visual detection of enzyme-linked antibodies in immunoassays: The liquid chromagen is added to the liquid buffer, mixed and is then ready for use.

Reader Service No. 100

Tel. (+1) 314-771-5750

Fax (+1) 314-771-5757

For more details, fill in the reader service card bound inside the journal.

\section{ERRATUM}

The company name was inadvertently omitted from Reader Service No. 98 in the November issue (Nature Medicine, 2, 1277; 1996). The ELISA kits for the determination of anti-BPI-IgG and anti-BPI-IgA antibodies are available from DLD Diagnostika. 